\title{
Análisis de Hábitos de Estudio en Alumnos de Primer Año de Ingeniería Civil Agrícola
}

\author{
L. Vidal ${ }^{1}$, M. Gálvez ${ }^{2}$ y L.B. Reyes-Sánchez ${ }^{3}$ \\ Universidad de Concepción, Facultad de Ingeniería Agrícola, (1) Departamento de Agroindustrias, \\ (2) Departamento de Riego y Drenaje, Casilla 537, Chillán-Chile \\ (e-mail: Ividal@udec.cl, mgalvez@udec.cl) \\ (3) Universidad Nacional Autónoma de México, Facultad de Estudios Superiores Cuautitlán, \\ Departamento de Química e Ingeniería Agrícola, Miramar No 54, 54740 Cuautitlán Izcalli, \\ México, D.F.-México (e-mail: Ibrs@servidor.unam.mx)
}

\begin{abstract}
Resumen
En este trabajo se analiza el problema del alto porcentaje de alumnos reprobados en los cursos del área de ciencias en la carrera de Ingeniería Civil Agrícola de la Universidad de Concepción en Chile. Para este estudio se seleccionaron y contrastaron dos encuestas que se aplicaron a cuatro grupos de estudiantes. Los resultados obtenidos indican que aparte de no tener los conocimientos básicos que requiere el curso, los estudiantes tampoco poseen hábitos y actitudes propias para el estudio. Todo esto no permite a los alumnos asimilar las materias y aprobar estos cursos. Se concluye que los mayores problemas que enfrentan los estudiantes, son la falta de técnicas adecuadas para leer y tomar apuntes, la inadecuada distribución de su tiempo, la falta de concentración y la falta de sitios adecuados para estudiar.
\end{abstract}

Palabras clave: enseñanza-aprendizaje, hábitos de estudio, educación universitaria, estrategias de aprendizaje

\section{Analysis of the Study Habits of Freshmen Students of Agricultural Engineering}

\begin{abstract}
An analysis was made of the problem of the high percentage of course failure in the science area within the agricultural engineering career of the University of Concepción in Chile. For this, two questionnaires were selected and contrasted after application to four groups of students. The results suggested that besides not having the basic concepts that a given course requires, the students did not have study habits and positive attitude to studying. All this prevent students to assimilate the subjects and pass the courses. It is concluded that the greatest problems confronted by the students are lack of reading and note-taking skills, inadequate programming of their time, failure to concentrate, and lack of adequate study areas.
\end{abstract}

Keywords: teaching-learning, study habits, university education, learning strategies

Nota: este artículo está tomado de "Información Tecnológica" [ISSN 0716-8756], vol. 13(2), 169-175 (2002) 


\section{INTRODUCCIÓN}

Debido a deficiencias en conocimientos previos y necesarios para las asignaturas de ciencias básicas (matemática, física, química y biología), que presentan los alumnos que ingresan a la carrera de Ingeniería civil agrícola, la Facultad de Ingeniería Agrícola realiza pruebas de diagnóstico en matemática; para conforme a los resultados obtenidos, ofrecerles cursos remediales en la materia que nivelen sus conocimientos en la misma.

Sin embargo, dado que la nivelación de conocimientos no implica la adquisición de hábitos y actitudes propias para el estudio y necesarias para el aprendizaje (Mager, 1964 y 1993); aún cuando se les ha implementado el curso remedial por lo menos durante 3 años, no se han observado cambios de actitud de los jóvenes frente al estudio (Zimbardo y Leippe, 1991). Por lo tanto, los resultados inmediatos que se esperaban obtener con la impartición de estos cursos a los alumnos, no han sido satisfactorios en términos de un incremento del número de aprobados en las asignaturas curriculares.

Siendo un hábito la repetición de una misma acción, y una actitud permanente que se desarrolla mediante el ejercicio y la voluntad (Pozo, 1989); hábito del estudio es definido por García et al. (1977), como la repetición del acto de estudiar realizado bajo condiciones ambientales del espacio, características y tiempo iguales. Dicho de otra forma, los hábitos y las actitudes propias para el estudio, se adquieren (Amestoy, 1993); siendo posible por tanto formar en los alumnos aquellos que no adquirieron durante sus estudios preuniversitarios, con la finalidad de mejorar su rendimiento escolar (Reyes-Sánchez y Obaya, 1998).

Este tema y problema de los hábitos y técnicas de estudio, lleva muchos años de discusión y sin embargo no es un tema ni un problema resuelto; lo que si está claro, es que el hábito para el estudio es el primero paso para activar y desarrollar la capacidad de aprender de los estudiantes (Alonso y Caturla, 1996) y de aquí la importancia de que el docente promueva la formación de éstos entre sus alumnos (Solar, 1999).

Por ello y con la finalidad de auxiliarlos adecuadamente en la adquisición de los hábitos y actitudes propias para el estudio, que les permitan obtener un mayor éxito escolar (Olshausky, 1976), se planteo como objetivo inicial el conocer cuantitativamente los hábitos de estudio que poseen los alumnos a su ingreso a esta carrera (Reyes-Sánchez y Obaya, 1998) en vez de partir de opiniones cualitativas y sin el respaldo de un estudio formal cuantitativo (Resnick, 1993; Quezada, 1981).

\section{METODOLOGÍA}

Para conocer los hábitos y actitudes propias para el estudio de los alumnos de la carrera de Ingeniería Civil Agrícola en el Campus Chillán de la Universidad de Concepción (Chile), fueron seleccionadas dos pruebas. El "Inventario de hábitos de estudio" de Bernstein (1997), en su versión Latinoamericana, que fue utilizado en un estudio anterior sobre el tema con alumnos de la carrera de ingeniería agrícola de la UNAM por Reyes-Sánchez y Obaya (1998) y la encuesta “¿Cómo estudias?” de Pérez y Cabrera (1994).

La encuesta "¿Cómo estudias?", consta de 33 preguntas clasificadas en seis ítems a evaluar (Fig.1) y fue aplicada a todos los alumnos de Química orgánica en el primer semestre del año 1999, así como a todos los de Química General y Cálculo I, durante el primer y segundo semestre del mismo año.

\begin{tabular}{|ll|}
\hline a) Lugar & d) En clase \\
b) Mesa & e) Tiempo de estudio \\
c) Silla & f) Método de trabajo \\
\end{tabular}

Fig. 1: Ítems para la encuesta “¿Cómo estudias?” 
28 a 33 puntos: Muy buen pronóstico para el estudio.

22 a 27 puntos: Pronóstico favorable.

16 a 21 puntos: Pronóstico poco favorable para el estudio.

0 a 15 puntos: Pronóstico muy poco favorable para el estudio.

Fig. 2: Interpretación de las puntuaciones obtenidas para la aplicación de la encuesta “¿Cómo estudias?". a) Técnicas para leer y tomar apuntes

b) Hábitos de concentración

c) Distribución del tiempo y relaciones sociales durante el estudio

d) Hábitos y actitudes generales de trabajo

Fig. 3: Ítems evaluados por la encuesta "Inventario de hábitos de estudio".

Cada pregunta tiene asignado un puntaje conforme a la respuesta dada y la interpretación de la puntuación total obtenida por el alumno en esta encuesta se muestra en la Fig. 2.

El "Inventario de hábitos de estudio" se aplicó al total de la promoción 2000, en la asignatura de Álgebra y trigonometría. Esta encuesta consta de 28 preguntas dispuestas en cuatro ítems Fig. 3.

De igual forma, también para esta encuesta cada pregunta tiene asignado un puntaje de acuerdo a la respuesta dada, siendo la sumatoria de todos ellos la puntuación total obtenida por el alumno e interpretándose como se muestra en la Fig.4.

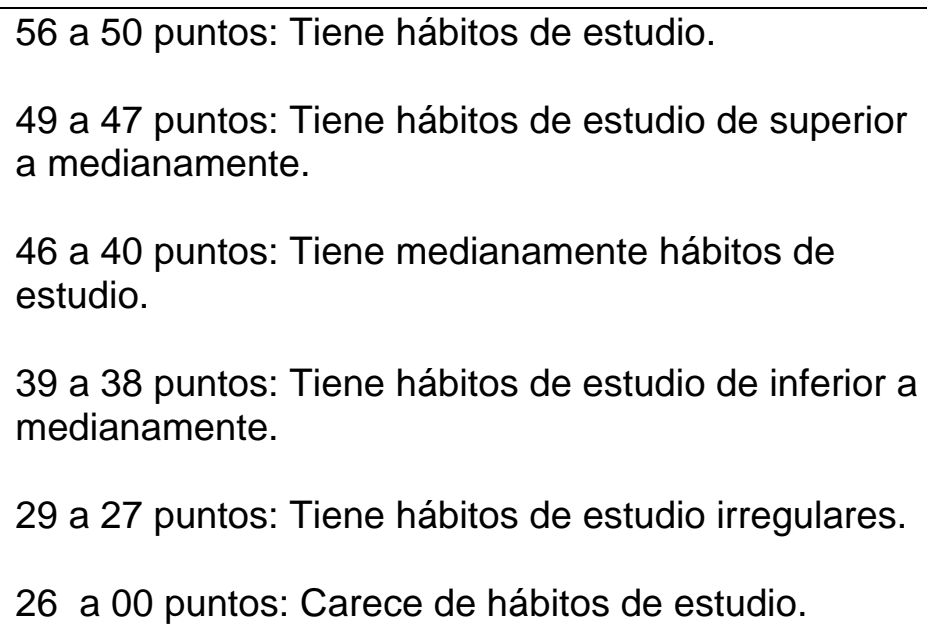

29 a 27 puntos: Tiene hábitos de estudio irregulares.

26 a 00 puntos: Carece de hábitos de estudio.

Fig.4: Interpretación de las puntuaciones obtenidas para el "Inventario de hábitos de estudio".

Durante la aplicación de ambas encuestas, se explicó a los alumnos el objetivo de este estudio, haciendo hincapié en la necesidad de que sus respuestas fueran el reflejo fiel de sus hábitos y actitudes para el estudio; así como su finalidad (Ajzen, 1988; Reyes-Sánchez y Obaya, 1998).

Finalmente, para cada uno de los cuatro grupos a los dos que se aplicaron las encuestas; se calcularon los porcentajes de frecuencia de respuesta para cada una de las secciones que las conforman, las puntuaciones promedio de los alumnos para ambos test y la interpretación correspondiente, contrastándola con las calificaciones promedio que obtuvieron en sus cursos. Los resultados se presentan a continuación.

\section{RESULTADOS Y DISCUSIÓN}

Del total de los 30 alumnos de Química orgánica a quienes se aplicó la encuesta "¿Cómo estudias?", solo 19 aprobaron el curso con una nota promedio de 59 (en una escala de 1 - 100), en tanto que la puntuación promedio obtenida por estos alumnos en la encuesta, fue de 19.11; 
misma que corresponde a un "Pronóstico poco favorable para el estudio" conforme a la escala en la Fig.2. y los porcentajes de frecuencia de respuesta de estos alumnos para los diferentes ítems de esta encuesta, se muestra en la Fig. 5.

\begin{tabular}{|c|c|}
\hline Ítems & $\begin{array}{c}\text { Resultados obtenidos en la encuesta “¿Cómo estudias?", } \\
\text { expresados en porcentaje. }\end{array}$ \\
\hline Lugar & $75 \%$ No tiene un lugar fijo para estudiar \\
\hline Mobiliario & $\begin{array}{l}28.6 \% \text { No percibe el concepto de orden asociado al estudio; } 17.9 \% \text { no dispone de } \\
\text { mobiliario adecuado; } 32.1 \% \text { no tiene asiento cómodo para ello. }\end{array}$ \\
\hline En clase & $61 \%$ Fotocopia apuntes de compañeros de clase. \\
\hline $\begin{array}{l}\text { Tiempo de } \\
\text { estudio }\end{array}$ & $\begin{array}{l}89.3 \% \text { No tiene control organizado del su tiempo ; } 64.3 \% \text { no prepara las clases en } \\
\text { forma continua; } 75 \% \text { presenta problemas de concentración. }\end{array}$ \\
\hline Método de trabajo & $\begin{array}{l}60.7 \% \text { Estudia a última hora; } 50 \% \text { no utiliza el diccionario; } 82 \% \text { no prepara sus } \\
\text { materias; } 25 \% \text { no revisa lo que escribe. }\end{array}$ \\
\hline $\begin{array}{l}\text { Autoevaluación } \\
\text { del alumno }\end{array}$ & $\begin{array}{c}64.3 \% \text { No tiene internalizado el hábito de estudio; } 14.3 \% \text { cree tenerlo; } 21.4 \% \text { dice no } \\
\text { saber que es método ni hábito de estudio. }\end{array}$ \\
\hline
\end{tabular}

Fig.5: Resultados obtenidos en la encuesta “¿Cómo estudias?” por alumnos de Química Orgánica.

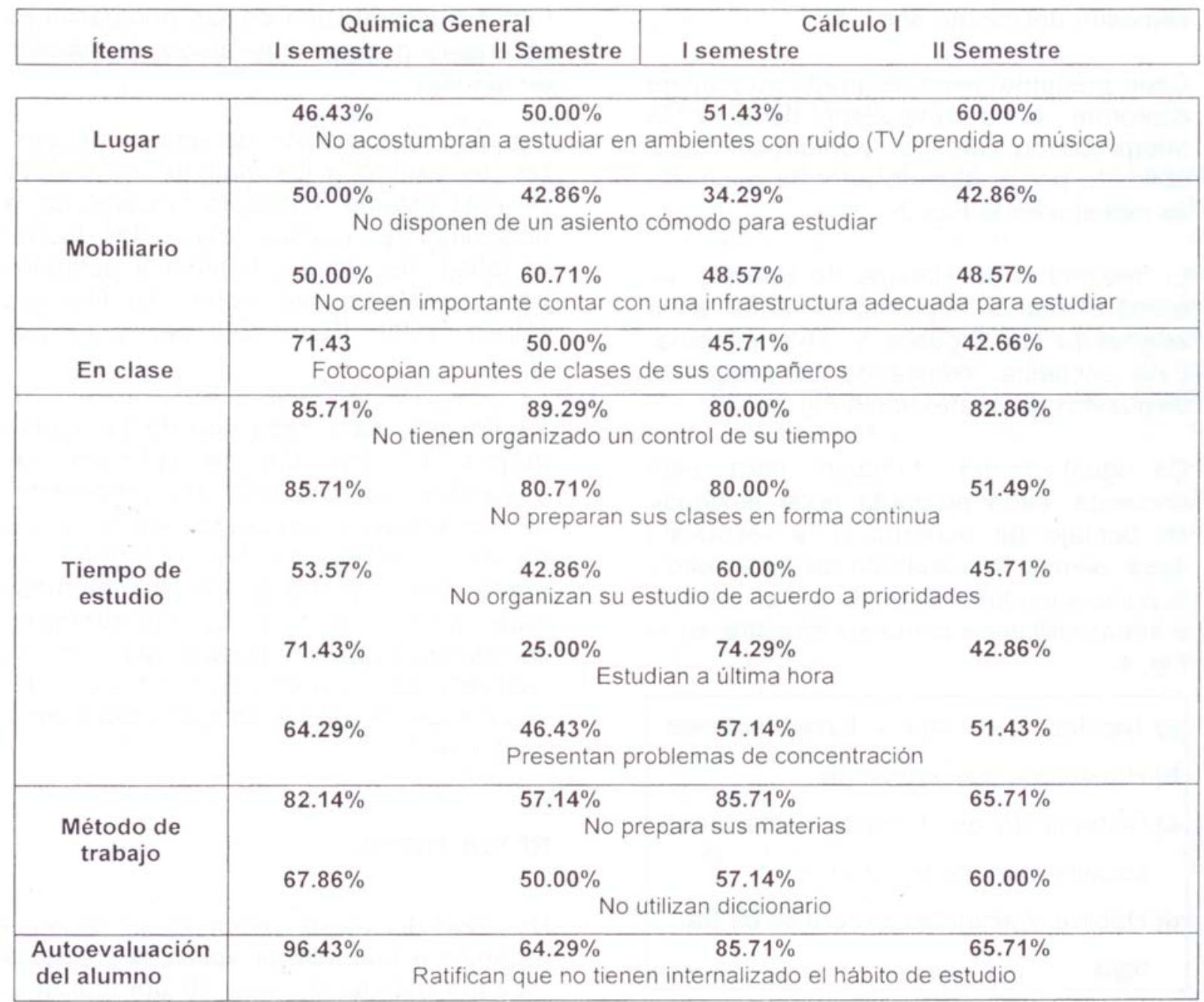

Fig. 6: Resultados obtenidos en la encuesta “¿Cómo estudias?”, por alumnos de química General y Cálculo I en dos semestres consecutivos. 


\begin{tabular}{|l|l|l|}
\hline Ítem & Puntaje promedio & Interpretación \\
\hline $\begin{array}{l}\text { Técnicas para leer y tomar } \\
\text { apuntes }\end{array}$ & 5.792 & $\begin{array}{l}\text { Técnicas de lectura y para } \\
\text { tomar notas deficientes }\end{array}$ \\
\hline hábitos de concentración & 4.685 & $\begin{array}{l}\text { Hábitos de concentración } \\
\text { deficientes }\end{array}$ \\
\hline $\begin{array}{l}\text { Distribución del tiempo y } \\
\text { relacione sociales durante } \\
\text { el estudio }\end{array}$ & 9.524 & $\begin{array}{l}\text { Inadecuada distribución del } \\
\text { tiempo }\end{array}$ \\
\hline $\begin{array}{l}\text { Hábitos y actitudes de } \\
\text { trabajo }\end{array}$ & 11.981 & Hábitos muy poco favorables \\
\hline
\end{tabular}

Fig.7: Resultados e interpretación para el "Inventario de hábitos de estudio".

Independientemente de cuál de las dos encuestas se aplicó a los alumnos, no hubo diferencia en el resultado obtenido que fue: "pronóstico poco favorable para el estudio" y no obstante haber impartido a los alumnos cursos remediales, los índices de reprobación no se modificaron ni reflejaron el esfuerzo realizado.

El análisis de los resultados mostrados en las figuras 5,6 y 7 indican que estos jóvenes no poseen técnicas adecuadas para leer y tomar apuntes y en promedio, el $85.43 \%$ dice no tener control sobre la distribución de su tiempo, el $58.85 \%$ del alumnado reconoce que presenta problemas de concentración, el $75 \%$ no tiene lugar fijo para estudiar, el $72.67 \%$ reconoce que no prepara sus clases en forma continua, el $54.86 \%$ dice que sólo estudia a última hora para sus exámenes y al hacerlo, el $54.16 \%$ se basa en fotocopias de los apuntes de quienes si asistieron a clase y tomaron notas. Llama la atención el hecho de que el $75.28 \%$ de estos alumnos están conscientes de ello y reconocen que no poseen los hábitos y actitudes propias ara el estudio que les permitan tener éxito en sus estudios.

Del análisis de los resultados obtenidos se desprende que tanto el número de reprobados, como las bajas calificaciones obtenidas en los cursos por los que si aprobaron; si bien es cierto que reflejan deficiencias en conocimiento previos, también lo es que el análisis de los resultados obtenidos en las encuestas evidencian que más allá de la enseñanza de los conocimientos básicos, los alumnos ciertamente también tienen un problema de ausencia de hábitos y actitudes propias para el estudio que no les permite asimilar y aprovechar estos cursos.

Por otro lado, los resultados académicos obtenidos en cursos tan diversos, además de guardar relación observable con los hábitos y actitudes propias para el estudio de que adolecen, evidencian lo difícil que les es organizar y desarrollar su propio proceso de aprendizaje en cualquier área del conocimiento sin estas herramientas; problema que no se resuelve con cursos de nivelación y deja clara la necesidad de implementar a la par un conjunto de estrategias que les permitan mejorar estos hábitos y por tanto, su rendimiento escolar (Christiensen et al., 1992; Zúñiga, 1993; Zúñiga, 1998; Reyes-Sánchez y Obaya, 1998).

Es importante señalar que coincidentemente, con el trabajo realizado sobre hábitos y actitudes de estudio con alumnos de la misma carrera de Ingeniería agrícola pero en la UNAM por ReyesSánchez y Obaya (1998); la falta de concentración en lo que estudian y los problemas de organización de su tiempo, son dos de los más graves problemas que enfrentan estos jóvenes y en ambos casos, un alto porcentaje de ellos estudia en ambientes con ruido.

\section{CONCLUSIONES}

Los resultados obtenidos permiten concluir que lo alumnos muestreados carecen de los hábitos y actitudes propias para el estudio que deberían haber adquirido durante su formación básica y preuniversitaria. La comparación de los datos obtenidos con los publicados para las áreas de la 
Ingeniería Agrícola en el estudio realizado en la UNAM, demuestran que en realidad, los alumnos ingresan a la universidad sin poseerlos.

Así mismo, del análisis de los resultados se concluye que no poseer técnicas adecuadas para leer y tomar apuntes, la inadecuada distribución de su tiempo y la falta de concentración, son en orden de prioridad sus mayores problemas; mismos que se agravan al no tener un sitio adecuado para estudiar ni considerar necesitarlo; siendo éste un punto de clara diferencia con los alumnos de la UNAM.

Es claro que la implementación de los cursos remediales, no lograron subsanar en los alumnos las deficiencias en conocimientos previos necesarios para las asignaturas de ciencias básicas, ni resolver la problemática del alto índice de reprobados; por lo que proponemos la conformación paralela de programas para modificar sus actitudes y hábitos frente al estudio, cuya adquisición se refleje en su rendimiento y en la eficiencia terminal.

\section{REFERENCIAS}

Ajzen, I; Attitudes, personality and behaviour, Stony Stratford Open University Press (1988).

Alonso J. y E. Catarla; La motivación en el Aula. PPC, Editorial y Distribuidora S.A., Madrid, España (1996).

Amestoy M.; El desarrollo de habilidades de pensamiento y su aplicación a la enseñanza. Centro Interuniversitario de desarrollo-CINDA. Fondo de desarrollo institucional del ministerio de educción Chile: 139-149 (1993).

Bernstein, J.; Inventario de hábitos de estudio de Gilbert Wrenn, Paidós (1997).

Christiensen, C.A., D.R., Massey y P.Y. Ysaac; Cognitive Strategies and Study Habits: An analysis of the easurement of Tertiary Students Learning. British Journal Educational Psychology: 61, 290299 (1992).

Garcia, C., M. Gutiérrez y E. Condemarin; A Estudiar, se Aprende. Metodología de Estudio Sesión por Sesión. Ediciones Universidad Católica de Chile (1997).

Mager, R.F.; Building an Instrument to measure behaviours and attitudes. Research Report: 143, Illions USA (1964).

Mager, R.F.; Creación de actitudes y aprendizaje. Marova, Madrid, España (1993).

Olshausky, J.E.; Reading as Problem Solving an Investigation of Strategies. Reading Research, Quaterly: 4, 15-27 (1976).

Pérez, E. y H. Cabrera; Orientación Tutorial, Ceis orientación, Santiago de Chile (1994).

Pozo, M.J.; Adquisición de estrategias de aprendizaje. Cuadernos de Pedagogía: 175, 8-11 (1989).

Quezada, C.R.; Los alumnos del bachillerato desean aprender a estudiar. Perfiles Educativos: 12 (1981).

Resnick. L.B.; Mathematics and Science Learning: a new conception. Science: 220, 477-478 (1993).

Reyes-Sánchez. L. y A. Obaya; Hábitos de Estudios de los Alumnos de Ingeniería Agrícola y su impacto en el rendimiento obtenido en un curso de Química Básica, Información Tecnológica: 9(5), 277-281 (1998). 
Solar, M.; Creatividad en Educación. Vicerrectoría Académica, Dirección de Docencia. Universidad de Concepción (1999).

Zimbardo, P. y M. Leippe; The psychology of attitude change and social influence, Mc. Graw Hill. N.Y., U.S.A. (1991).

Zúñiga, M.C.; Estilos y estrategias de aprendizaje de los alumnos de la Universidad de La Serena y su relación con la percepción del contexto académico. Centro Interuniversitario de DesarrolloCINDA. Fondo de Desarrollo del Ministerio de Educación-Chile: 159-166 (1993).

Zúñiga, M.C.; Algunos criterios para la formulación de una estrategia integral de docencia en educación superior: una mirada desde la relación enseñanza-aprendizaje. Centro interuniversitario de Desarrollo-CINDA. Fondo de Desarrollo del Ministerio de Educación-Chile: 114-126 (1998). 